PAPER

\title{
Psychosocial outcomes at 18 months after good neurological recovery from aneurysmal subarachnoid haemorrhage
}

\author{
J Powell, N Kitchen, J Heslin, R Greenwood
}

J Neurol Neurosurg Psychiatry 2004;75:1119-1124. doi: 10.1136/innp.2002.000414

See end of article for authors' affiliations .....................

Correspondence to: Dr J Powell, Goldsmiths College, Lewisham Way, New Cross, London, SE14 6NW, UK; i.powell@ gold.ac.uk

Received 25 July 2002

In revised form

8 August 2003

Accepted

10 September 2003 Objectives: To evaluate functioning, 18 months after surgery, of 49 patients with good neurological
recovery following aneurysmal subarachnoid haemorrhage (SAH), and to determine the extent of any improvements in disturbances of mood, cognitive functioning, and levels of activity and participation previously observed at 9 month follow up.

Methods: SAH patients, matched for age, gender, and occupation with healthy control participants, completely quantitative measures of mood (HADS, FIES, BDI) and activity/participation (BICRO-39 scales), and a brief cognitive assessment battery (verbal fluency, digit span, prose recall). Controls completed the HADS and the BICRO-39.

Results: Patients showed some recovery of cognitive functioning, though impairments of prose recall persisted. Anxiety and depression symptoms were higher in patients than in controls, but fewer than $20 \%$ scored in the clinical range on any questionnaires except for RIES-Intrusive thoughts (22\%); only three showed signs of full blown post-traumatic stress disorder. Almost half showed elevated dependence on others for domestic activities and organisation and abnormally low levels of employment. Very little variance in outcome was predicted by demographic variables, neurological or cognitive impairment, prior life stress, or mood. However, levels of social activity and self-organisation were related to persisting fatigue.

Conclusions: The observed decline in intrusive thoughts and avoidance over time is consistent with that seen after life threatening illness or trauma. The persistent reductions in independence and levels of employment may in some cases reflect considered lifestyle adjustments rather than adverse and unwanted changes but in others indicate a need for focused rehabilitation.
W e recently described 9 month follow-up results from a prospective study of 52 patients with good neurological recovery after surgery for aneurysmal subarachnoid haemorrhage (SAH). ${ }^{1}$ Participants were assessed on self-report measures of emotional symptomatology (the Hospital Anxiety and Depression Scale (HADS), the Beck Depression Inventory (BDI), the Revised Impact of Events Scale (RIES)) and of activity/participation (the BICRO-39 scales), and on cognitive tests (verbal fluency, prose recall, digit span).

Rates of clinically significant HADS anxiety and depression scores $(>10)$ were two to three times higher in the SAH group than in a closely matched healthy control group, confirming reports elsewhere of elevated psychiatric morbidity after SAH. ${ }^{2}{ }^{3}$ High levels of intrusive thoughts about the illness and/or hospitalisation were reported by over $25 \%$ of patients, and $17 \%$ reported avoidance behaviour at a level commensurate with that seen in post-traumatic stress disorder (PTSD). ${ }^{4}$ Cognitively, although there was no evidence of impairments in digit span or verbal fluency, nearly a third of patients scored below the 10th percentile on a test of immediate prose recall. Other research groups have likewise reported neuropsychological dysfunction in some patients with apparently good neurological outcomes. ${ }^{5-8}$

Most striking, however, were patients' reduced levels of activity and participation: over 35\% showed abnormally low levels (below the 10th percentile of the matched control group) of independence on the Mobility scale of the BICRO39 (extended activities of daily living such as laundry, shopping, etc), whilst $50 \%$ reported similarly low levels of independence in Self-Organisation (for example, planning, structuring domestic/personal routines, managing finances). Only 44\% had resumed "normal" levels of Productive Employment (that is, engagement in paid or voluntary employment, education/training, or childcare). Whilst other research groups have noted similarly low rates of resumption of former employment a year or more after surgery, ${ }^{9}{ }^{10}$ and have described a high rate of subjective complaints of headaches and fatigue ${ }^{11}{ }^{12}$ with associated reductions in leisure and social activities, ${ }^{5}$ the increased level of dependence on others of this neurologically unimpaired group for routine domestic activities has not previously been identified using quantitative measures.

There were only slight improvements on the BICRO-39 from 3 to 9 months, reaching statistical significance only for the Mobility scale. Neither independence nor employment were predicted by a set of demographic variables, indices of premorbid health, and indices of early clinical outcome (GOS scores, dysphasia), consistent with other reports. ${ }^{13}$ Current mood disturbance was significantly associated with only one BICRO scale (Self-Organisation).

The present paper describes the functioning of the same patient group at 18 months post-discharge.

\footnotetext{
Abbreviations: BDI, Beck Depression Inventory; BICRO-39, Brain Injury Community Rehabilitation Outcome - 39; COWAT, Controlled Oral Word Association Test; HADS, Hospital Anxiety and Depression Scale; LCS, Life Change Scale; MANOVA, multivariate analyses of variance; PTSD, post-traumatic stress disorder; RIES, Revised Impact of Events Scale; RLCQ, Revised Life Changes Questionnaire; SAH, subarachnoid haemorrhage
} 


\section{METHODS \\ Design}

This was a prospective study of 52 consecutive patients admitted to the neurovascular service of the National Hospital for Neurology and Neurosurgery (NHNN) for treatment of aneurysmal SAH, and making a good neurological recovery. Patients were interviewed at hospital discharge and then assessed 3, 9, and 18 months later on measures of cognitive and psychosocial functioning. The study aimed (i) to determine the prevalence of various cognitive, affective, and psychosocial problems at each follow-up point, and thus to trace the pattern of recovery over time, and (ii) to determine whether adverse outcomes could be predicted from early clinical signs or pre-injury characteristics. Ethical approval for the study was given by the hospital Ethics Committee.

For each participant a healthy control of the same gender, closely matched for age, ethnicity, and occupational status immediately prior to the SAH, completed, once only, the HADS and the BICRO-39. For these variables, the scores of the SAH participants at each follow-up point were compared with those of this reference group, which provided better matching for demographic factors than could be achieved from the limited existing normative data. Patients' scores on the remaining measures were referenced to published norms for healthy adults of similar age.

\section{SAH participants}

All patients were aged 16 or over, had a good initial WNFS grade (I or II, that is, no motor deficits, and Glasgow Coma Scale scores of 15 or 14, respectively), and had made a good neurological recovery at the point of hospital discharge (GOS score of 4 or 5). All participants gave informed consent to follow up by an independent assessor $(\mathrm{JH})$ either at the hospital or in their own homes according to their preference.

\section{Assessments}

Fuller details of the measures described below are given in our earlier paper. ${ }^{1}$

\section{Neurological impairment}

The presence of sensorimotor symptoms possibly attributable to the SAH or surgery was assessed 3 months after discharge using the Bond Neurophysical Scale. ${ }^{14}$

\section{Stressful life events}

The Revised Life Changes Questionnaire (RLCQ) ${ }^{15}$ required respondents to indicate whether, over the 12 months preceding their SAH, they had experienced each of 74 objectively defined life events. Scores are represented as total "life change units".

Patients also completed the 10-item Life Change Scale $(\mathrm{LCS})^{16}$ which asked them to rate in global terms, on 0-10 scales, the subjective extent of stress in each of ten areas of their life (for example, finance, relationships, work) over the same period.

\section{Tests of cognitive function}

Digit Span, ${ }^{17}$ the Controlled Oral Word Association Test (COWAT), ${ }^{18}$ and a test of prose recall ${ }^{20}$ similar to the WMS Logical Memory test but with four equivalent versions, were administered using parallel forms at each assessment occasion. Verbal fluency and digit span scores were converted to age-related percentiles using published norms. ${ }^{17}{ }^{19}$ For the prose recall test, immediate recall scores below $7 / 25$ and delayed recall scores below $4 / 25$ fall below the 10th percentile. $^{120}$

\section{Mood state and fatigue}

Symptoms of anxiety and depression were assessed using the Hospital Anxiety and Depression Scale (HADS), ${ }^{21}$ the clinical cut off for both mood states being 10/11 (that is, scores of $11+$ fall within the clinical range). The Beck Depression Inventory $(B D I)^{22}$ was also used; here, scores of 19 or higher fall outside the normal range.

The Revised Impact of Events Scale (RIES) ${ }^{4}$ yielded indices of intrusive thoughts about the SAH and avoidance of reminders/emotional reactions; the scale serves as a screening (though not a diagnostic) instrument for PTSD. Cut offs of 11/12 for Intrusions and 13/14 for Avoidance have been used to categorise scores as "abnormal". ${ }^{14}$

Participants also rated their current experience of fatigue, defined as physical and/or mental tiredness, using a simple three point scale $(0=$ none, $\mathrm{l}=$ mild to moderate, $2=$ severe) .

\section{Social participation}

The Brain Injury Community Rehabilitation Outcome - 39 (BICRO-39) scales $^{23}$ measure the impact of brain injury on domestic, social, and psychological functioning in patients living in the community. The scales employed here were Personal Care, Mobility, Self-Organisation, Socialising, Productive Employment (including voluntary and paid work, childcare, and education/training), and Psychological WellBeing; in each case scores can range from 0 to 5 , with 0 indicating good outcome (for example, independence, high level of activity) and 5 poor outcome (for example, dependence, low engagement, poor psychological state).

\section{Statistical analyses}

To test the prediction that in general SAH patients would show more severe problems than demographically matched healthy adults, two multivariate analyses of variance (MANOVAs) were used to compare the 18 month scores of the SAH group with those of the control group on (i) the two HADS subscales and (ii) the six BICRO subscales.

Repeated measures MANOVAs were used to determine whether the SAH group showed significant recovery from 9 to 18 months on: (a) the five cognitive measures; (b) four of the mood measures (HADS and RIES subscales); and (c) the six BICRO scales. For completeness, univariate statistics are also reported from these MANOVAs for the individual component measures, with Bonferroni corrections for multiple comparisons within each family of measures.

Multiple regression was used to explore the relationships between a number of predictor variables and psychosocial outcomes.

\section{RESULTS}

\section{Participants}

Both SAH and control groups comprised 35 women and 17 men. SAH participants were aged from 27 to 82 years (mean (SD) age 46.9 ( 10.4 ) years) and controls from 26 to 74 years (mean (SD) age 46.7 (10.7) years). As detailed in our earlier paper, ${ }^{1}$ the groups were very comparable for occupational status.

Of the SAH patients, 47 had their aneurysms clipped, four had them coiled or wrapped, and one was managed conservatively. A total of 44 had a discharge GOS score of 5; the remainder scored 4 . Of the participants, 51 were successfully contacted and assessed 3 months after hospital discharge, 48 at 9 months, and 49 at 18 months. The 49 participants whose 18 month results are reported here comprised 33 women and 16 men, with a mean (SD) age of 46.0 (10.0) years.

At 3 months, the Bond Neurophysical Scale indicated that $23.5 \%$ of participants had impaired visual acuity (not 
necessarily due to the SAH), $8 \%$ a visual field deficit, $6 \%$ diplopia, $15.7 \%$ tinnitus, $27.5 \%$ dysphasia, and $12 \%$ vertigo. None suffered from nystagmus, ataxia, or locomotory deficits. In all, $61 \%$ of the sample presented with at least one of the above symptoms, though in most cases they were mild.

Eight SAH participants reported a prior history of treatment for psychiatric or emotional problems; two of these, and another two participants, also reported prior drug or alcohol problems. Very few reported significant histories of physical ill health, though one had had a head injury necessitating hospital admission, and one had suffered a previous CVA (cerebrovascular accident).

\section{Changes in cognitive and psychosocial dysfunction from 9 to 18 months post-SAH}

Table 1 shows (i) mean scores on the measures of cognitive functioning, mood, and participation in the SAH participants at 9 and 18 months, (ii) healthy control scores on the HADS and BICRO (measured once only), and (iii) prevalence of "abnormality", operationally defined either as above clinical cut offs (for mood measures), below the 10th percentile when compared with published normative data (cognitive measures), or above the 90th percentile of the matched control group (BICRO scales).

\section{Cognitive functioning}

SAH patients' scores on these variables were approximately normally distributed. Reflecting the pattern seen at 9 months, only on immediate prose recall was there an elevated rate of low scores ( $<10$ th percentile): at $22 \%$, this represents some recovery from the $34 \%$ impairment rate at 9 months but is still about twice the expected rate in neurologically intact individuals. A total of $13.6 \%$ scored below the 10th percentile on delayed recall, barely more than would be expected in the general population.

For the 43 SAH patients with complete data on all measures, MANOVA revealed significant improvement from 9 to 18 months $\left(\mathrm{F}_{5,38}=9.0, \mathrm{p}<0.001\right)$. Univariate ANOVAs on the five tests individually, utilising data from all participants who had completed each test on both occasions and with Bonferroni correction reducing the probability level for statistical significance to 0.01 , showed significant improvements on three (verbal fluency, $F_{1,42}=11.7$, $\mathrm{p}<0.001$; delayed recall, $\mathrm{F}_{1,42}=8.0, \mathrm{p}<0.01$; forwards digit span, $\left.F_{1,43}=9.2, p<0.005\right)$, and trends for immediate prose recall $\left(\mathrm{F}_{1,42}=6.2, \quad \mathrm{p}=0.02\right)$ and reversed digit span $\left(\mathrm{F}_{1,43}=5.5, \mathrm{p}<0.03\right)$.

\section{Mood state and fatigue}

All indices were reasonably normally distributed. As shown in table 1, only the two RIES subscales showed recovery from 9 to 18 months. Nevertheless, approximately $22 \%$ continued to score in the clinical range for Intrusions (down only slightly from $25.5 \%$ at 9 months) and $13 \%$ in the clinical range for avoidance (again, only a little lower than the 17\% at 9 months). The proportions scoring in the clinical range on the BDI, HADS Anxiety, and HADS Depression were all marginally higher at 18 than at 9 months.

MANOVA comparing the 18 month HADS scores of the SAH patients against those of the control group showed a weak trend towards elevated scores in the SAH group $\left(\mathrm{F}_{2,47}=2.26, \mathrm{p}<0.11\right)$. Univariate comparisons indicated a stronger trend for $\mathrm{SAH}$ patients to be more anxious than controls $\left(\mathrm{F}_{1,48}=4.44, \mathrm{p}<0.05\right)$ but no difference in depression.

A MANOVA on the two HADS and two RIES subscales revealed a trend for the SAH patients to show improvement from 9 to 18 months $\left(\mathrm{F}_{4,40}=2.1, \mathrm{p}=0.10\right)$. Univariate ANOVAs, with Bonferroni correction of the significance level to $\mathrm{p}<0.01$, detected significant recovery on the RIES Intrusions subscale $\left(\mathrm{F}_{1,43}=7.5, \mathrm{p}<0.01\right)$, a trend on RIES Avoidance $\left(F_{1,44}=4.2, p=0.05\right)$, but no change on HADS Anxiety or Depression or on the BDI ( $\mathrm{F}<1, \mathrm{NS}$, in all cases).

Fatigue was a common problem, being reported as moderate by 30 and severe by nine participants at 9 months; there was no significant reduction in mean scores by 18 months, with 32 still reporting it as moderate and five as severe $\left(\mathrm{F}_{1,47}=2.4\right.$, NS).

\section{Participation: the BICRO-39}

Although scores on several of the BICRO-39 subscales were non-normally distributed, ANOVA is relatively robust with

Table 1 Cognitive, mood, and psychosocial function data for SAH participants at 9 and 18 months and for matched controls

\begin{tabular}{|c|c|c|c|c|c|c|c|c|c|c|c|c|}
\hline & \multicolumn{4}{|c|}{ Controls } & \multicolumn{4}{|c|}{ SAH group at 9 months } & \multicolumn{4}{|c|}{ SAH group at 18 months } \\
\hline & $\mathbf{n}$ & $\overline{\mathbf{x}}$ & (SD) & $\begin{array}{l}\text { Percentage in } \\
\text { clinical range* or } \\
<10 \text { th percentile } t\end{array}$ & n & $\overline{\mathbf{x}}$ & (SD) & $\begin{array}{l}\text { Percentage in } \\
\text { clinical range* or } \\
<10 \text { th percentilet }\end{array}$ & $\mathbf{n}$ & $\overline{\mathbf{x}}$ & (SD) & $\begin{array}{l}\text { Percentage in } \\
\text { clinical range* or } \\
<10 \text { th percentilet }\end{array}$ \\
\hline \multicolumn{13}{|l|}{ Cognitive measures } \\
\hline Verbal fluency $\ddagger$ & & & & & 44 & 56.0 & (32.7) & $11.6 \%$ & 44 & 65.1 & (30.7) & $11.4 \%$ \\
\hline Prose recall-immediate (0-24) & & & & & 44 & 9.3 & (3.9) & $34.1 \%$ & 44 & 10.3 & $(3.6)$ & $22.7 \%$ \\
\hline Prose recall-delayed (0-24) & & & & & 44 & 8.3 & $(4.0)$ & $18.2 \%$ & 44 & 9.4 & $(4.1)$ & $13.6 \%$ \\
\hline Digit Span Forwards $\ddagger$ & & & & & 45 & 65.9 & $(30.6)$ & $4.4 \%$ & 46 & 73.7 & $(25.8)$ & $4.3 \%$ \\
\hline Digit Span Backwardsł & & & & & 45 & 54.0 & (30.4) & $8.9 \%$ & 46 & 62.5 & (31.7) & $8.7 \%$ \\
\hline \multicolumn{13}{|l|}{ Mood measures } \\
\hline HADS Anxiety (0-21) & 52 & 5.3 & $(3.0)$ & $5.7 \%$ & 47 & 7.3 & $(4.2)$ & $17.0 \%$ & 49 & 7.2 & (3.9) & $18.4 \%$ \\
\hline HADS Depression (0-21) & 52 & 3.8 & (3.5) & $3.8 \%$ & 47 & 5.0 & (3.6) & $8.5 \%$ & 49 & 5.1 & (3.6) & $10.2 \%$ \\
\hline RIES - Intrusions $(0-35)$ & & & & & 47 & 8.5 & (7.0) & $25.5 \%$ & 46 & 6.6 & (5.5) & $21.7 \%$ \\
\hline RIES-Avoidance (0-40) & & & & & 47 & 6.9 & (6.7) & $17.0 \%$ & 47 & 5.7 & (5.3) & $12.8 \%$ \\
\hline BDI (0-63) & & & & & 44 & 9.2 & (6.9) & $11.4 \%$ & 49 & 9.4 & (7.3) & $16.3 \%$ \\
\hline \multicolumn{13}{|l|}{ Participation (BICRO) } \\
\hline Personal Care (0-5) & 52 & 0.0 & $(0.2)$ & & 48 & 0.0 & $(0.0)$ & $2.1 \%$ & 49 & 0.0 & (0.1) & $4.1 \%$ \\
\hline Mobility (0-5) & 52 & 0.2 & (0.3) & & 48 & 0.7 & (1.1) & $35.4 \%$ & 49 & 0.7 & $(1.0)$ & $42.9 \%$ \\
\hline Self-Organisation (0-5) & 52 & 0.1 & $(0.4)$ & & 48 & 0.8 & (1.3) & $50.0 \%$ & 49 & 0.8 & $(1.0)$ & $46.9 \%$ \\
\hline Socialising $(0-5)$ & 52 & 2.9 & $(0.7)$ & & 48 & 2.9 & (0.8) & $9.3 \%$ & 49 & 3.1 & (0.8) & $16.3 \%$ \\
\hline Employment $(0-5)$ & 52 & 3.0 & $(0.8)$ & & 48 & 4.0 & $(0.9)$ & $56.2 \%$ & 49 & 4.0 & $(0.9)$ & $49.0 \%$ \\
\hline Psychological Well-being (0-5) & 52 & 1.5 & $(0.7)$ & & 48 & 1.8 & $(0.8)$ & $12.5 \%$ & 49 & 1.9 & $(0.8)$ & $12.1 \%$ \\
\hline
\end{tabular}


Table 2 Relationships between background (clinical, demographic, pre-SAH) data and selected psychosocial outcome measures

\begin{tabular}{|c|c|c|c|c|c|c|c|c|c|}
\hline & \multirow[b]{2}{*}{ n† } & \multicolumn{6}{|c|}{ BICRO-39 scales } & \multirow{2}{*}{\multicolumn{2}{|c|}{$\begin{array}{l}\text { Mood Disturbance } \\
\text { (HADS+RIES) } †\end{array}$}} \\
\hline & & \multicolumn{2}{|c|}{ Productive Employment } & \multicolumn{2}{|l|}{ Mobility } & \multicolumn{2}{|c|}{ Self-Organisation } & & \\
\hline \multicolumn{10}{|l|}{ Demographic characteristics } \\
\hline Age & 49 & $r=0.32$ & * & $r=0.06$ & NS & $r=-0.06$ & NS & $r=-0.12$ & NS \\
\hline Gender & 49 & $Z=-0.70$ & NS & $Z=-1.47$ & NS & $Z=-0.24$ & NS & $Z=-0.92$ & NS \\
\hline \multicolumn{10}{|l|}{ Clinical presentation } \\
\hline Glasgow Outcome Scale score & 49 & $Z=-1.72$ & NS & $Z=-1.93$ & 0.06 & $Z=-0.46$ & NS & $Z=-0.12$ & NS \\
\hline Dysphasia at 3 months & 49 & $Z=-2.10$ & * & $Z=-2.40$ & $*$ & $Z=-2.56$ & ** & $Z=-1.60$ & NS \\
\hline Immediate prose recall at 3 months & 43 & $r=-0.37$ & ** & $r=-0.12$ & NS & $r=-0.12$ & NS & $r=-0.13$ & NS \\
\hline \multicolumn{10}{|l|}{ Prior history } \\
\hline Life stress (objective)-RLCQ & 49 & $r=-0.03$ & NS & $r=-0.24$ & NS & $r=-0.05$ & NS & $r=0.12$ & NS \\
\hline Life stress (subjective) - LCU & 49 & $r=0.13$ & NS & $r=0.10$ & NS & $r=0.19$ & NS & $r=0.22$ & NS \\
\hline Health problems past year & 49 & $r=0.25$ & NS & $r=0.38$ & ** & $r=0.12$ & NS & $r=-0.04$ & NS \\
\hline Mental health treatment (ever) & 46 & $Z=-0.07$ & NS & $Z=-0.72$ & NS & $Z=-0.70$ & NS & $Z=-2.00$ & * \\
\hline
\end{tabular}

respect to skewness and so the statistical approach is the same as for the other variables.

Table 1 shows that at both 9 and 18 months close to half of the SAH participants scored abnormally (above the 90th percentile of the control participants, indicating low activity/ participation) on Self-Organisation and Productive Employment. Over a third likewise scored abnormally on the Mobility subscale at both follow-up points.

Although the scores indicate slight improvements in SelfOrganisation and Productive Employment, and increasing problems with Mobility, Personal Care, and Socialising, MANOVA revealed there to be no significant change either overall $\left(\mathrm{F}_{6,42}=1.6, \mathrm{NS}\right)$ or on any individual scale $\left(\mathrm{F}_{1,47}<4.0\right.$, $\mathrm{p}>0.05$ in all six cases).

The 18 month BICRO scores of the SAH group were significantly higher than control group scores (MANOVA: $\left.\mathrm{F}_{6,43}=10.5, \mathrm{p}<0.001\right)$. Univariate ANOVAs, with Bonferroni correction of $\mathrm{p}$ to $<0.008$, showed significantly lower independence/participation in the SAH group relative to the control group on Mobility, Self-Organisation, and Productive Employment $\left(\mathrm{F}_{1,48}>16, \mathrm{p}<0.001\right.$ in each case $)$, and a trend towards poorer Psychological Well-Being $\left(\mathrm{F}_{1,48}=6.4\right.$, $\mathrm{p}=0.015$ ); however there were no between-group differences for Personal Care or Socialising $\left(\mathrm{F}_{1,48}<1.5\right.$, NS, for both).

\section{Predictors of 18 month outcomes}

The relatively small sample size of this study meant that it had sufficient power to explore relationships between only a limited number of 18 month outcome variables and potential predictors. Within the functional outcomes, three were of particular interest because they demonstrated a high level of sensitivity to adverse change: BICRO Productive Employment, Mobility, and Self-Organisation. Since these scales are not highly intercorrelated, ${ }^{23}$ they were considered separately.

Intercorrelations between the mood-related indices (the four RIES and HADS subscales), however, were all highly significant $(\mathrm{p}<0.001)$ and so a total "mood disturbance" score was computed by summing these component scores.

Nine predictors were selected as follows:

- demographic variables: (a) age and (b) gender

- indicators of clinical impairment: (a) discharge GOS score (4 or 5); (b) dysphasia at 3 months (present/absent); (c) immediate prose recall at 3 months (raw score)

- indicators of pre-SAH health and life stress: (a) physical health in preceding 12 months, rated from 1 (good) to 4 (major problems); (b) history of any treatment for mental health problems (yes/no); (c) RLCQ scores (objective stressors) for the year preceding SAH; (d) LCS scores (subjective stress) for year preceding SAH.

Other potential predictors were excluded either because they showed insufficient variance or because a related index was selected in preference (for example, immediate prose recall was taken as a marker of cognitive impairment because it was more likely than other cognitive indices to be impaired).

Table 2 shows the individual relationships between each of the nine predictors and each of the four outcome variables, with non-parametric correlations used for continuous variables (age, physical health, prose recall, RLCQ, and LCS) and Mann-Whitney U tests for dichotomous variables (gender, dysphasia, GOS scores, and mental health).

All nine predictor variables were entered simultaneously into separate multiple regressions on each of the four outcome variables. The sample size (up to 44 participants with complete data for each multiple regression) is a little below below the recommended minimum for this technique $(40+p$, where $p$ represents the number of predictor

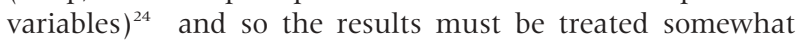
cautiously.

The nine predictors jointly accounted for less than $7 \%$ of the variance in BICRO Self-Organisation $(R=0.39$; adjusted $\mathrm{R}^{2}=-0.067 ; \mathrm{F}_{9,34}<1.0$, NS). For BICRO Mobility, adjusted $\mathrm{R}^{2}$ was $0.35 \quad\left(\mathrm{R}=0.70 ; \mathrm{F}_{9,34}=3.5, \mathrm{p}<0.05\right)$; the only individual predictor to account for a significant unique portion of the variance (that is, after partialling out the other predictors) was prior physical health (part correlation $=0.40 ; \mathrm{B}=3.16, \mathrm{p}<0.002$ ). Only $19 \%$ of the variance in BICRO Productive Employment was predicted by the model, and this fell just short of significance $\left(\mathrm{F}_{9,34}=2.1, \mathrm{p}=0.06\right)$; no individual predictor showed part-correlations even approaching significance ( $\mathrm{p}>0.08$ in all cases).

Finally, $20 \%$ of the variance in mood disturbance was predicted by this set of variables $(\mathrm{R}=0.61$; adjusted $\left.\mathrm{R}^{2}=0.20\right)$, though this did not quite achieve statistical significance $\left(F_{9,31}=2.1, p=0.06\right)$. Two predictors made significant unique contributions to the variance: prior mental health (part correlation $=0.39$, that is, $16 \%$ of variance; $\mathrm{B}=17.5 ; \mathrm{p}<0.01$ ); and dysphasia (part correlation $=0.30$, that is, $9 \%$ of variance; $\mathrm{B}=12.2 ; \mathrm{p}<0.05$ ).

\section{Relationships between emotional and functional outcomes}

Mood (sum of HADS and RIES scores) at 18 months was not significantly correlated with 18 month scores on either BICRO Mobility, Self-Organisation, or Productive 
Employment $(\mathrm{r}<0.35, \mathrm{p}>0.09$ in every case $)$. Subjective fatigue ratings, by contrast, were associated with functional outcomes. The 37 participants who reported fatigue as moderate or severe at 18 months showed lower independence in Self-Organisation and lower levels of Socialising than the 12 who did not (Mann-Whitney tests: $Z=-3.1$ and $-2.4, \mathrm{p}<0.02$, respectively). They also tended to be less independent in Mobility $(\mathrm{Z}=-1.9, \mathrm{p}=0.06)$, though their scores on Personal Care and Productive Employment did not differ from the non-fatigued participants $(Z<1$, NS, in both cases).

\section{DISCUSSION}

At 9 months after these patients had made a good neurological recovery from surgery for aneurysmal SAH, about a third showed evidence of cognitive impairment which was particularly manifest on a measure of verbal recall, ${ }^{1}$ consistent with findings from some other empirical studies. ${ }^{5-8}$ The slight improvements in verbal recall over the ensuring 9 months fell just short of significance, with over $20 \%$ of patients continuing to score below the 10th percentile. This is approximately twice the expected ("normal") rate. However, no impairment was evident on tests of verbal fluency, digit span, or delayed prose recall, tests selected because previous research had shown them to be sensitive to post-SAH dysfunction.

Thus, the majority of participants were functioning within normal limits on most cognitive tests 18 months after surgery. Indeed, of those who completed all five tests, only $27 \%$ scored below the 10th percentile on one or more tests and only 9\% scored below this level on two or more tests. Allowing for normal measurement error in cognitive testing, an expected "abnormality" rate of $10 \%$ for each test individually, and individual variation in cognitive abilities, these patterns of abnormal performance are only slightly higher than would be found within the general population. These findings are consistent with at least two previous studies which have found little indication of cognitive dysfunction in similar patients a year after surgery. ${ }^{25} 26$

It is striking, given the absence of marked cognitive impairment or physical disability in the majority of these patients, that $40-50 \%$ reported abnormally high dependence on others for assistance with extended activities of daily living and self-organisation, and low levels of engagement in productive employment-related activities including paid and unpaid work, education or training, and childcare.

There was remarkably little improvement in psychosocial functioning from 9 to 18 months post-surgery, and neither dependence on others for organisation nor low levels of productive employment were significantly predicted by any of nine demographic, clinical/cognitive outcome, and pre-SAH stress/health-related variables. The absence of any significant predictors of independence in Self-Organisation failed to mirror the findings at 9 months, ${ }^{1}$ when memory impairment at 3 months and prior life stress (RLCQ scores) both emerged as independent predictors. These findings suggest that once increased levels of dependence and altered patterns of activity have been established for several months, they become entrenched and are relatively resistant to further spontaneous change. This may partly reflect the observations made by some patients ${ }^{1}$ that their experience of lifethreatening illness had prompted them to reappraise their life priorities and adjust their lifestyles accordingly.

The nine predictors did, however, jointly explain $35 \%$ of the variance in Mobility (independence in physical activities inside and outside the home) at 18 months compared with $20 \%$ at 9 months. Contrasting with the lack of individually significant predictors at 9 months, by 18 months $16 \%$ of the variance was attributable uniquely to pre-SAH physical health. Although it is unclear why the role of prior physical health should become apparently more influential, it might possibly be associated with the development of additional medical problems which then play a role in perpetuating dependence on others for domestic activities. This speculative explanation cannot be directly tested here, but in future research it would be of interest to record the occurrence of other health-related problems after SAH.

Mood state generally showed only a trend towards improvement from 9 to 18 months, though there was a significant reduction in intrusive thoughts relating to the illness and hospitalisation. Although over $20 \%$ of patients continued to report frequent intrusive thoughts, only three (6\%) scored above clinical cut offs on the RIES for both intrusive thoughts and avoidance. This suggests that clinical diagnoses of post-traumatic stress would be uncommon at this stage post-illness. The temporal decline in the rates of patients reporting both symptoms, from $30 \%$ at 3 months to $15 \%$ at 9 and then $6 \%$ at 18 months, is similar to the pattern for post-traumatic symptomatology after other life-threatening events. ${ }^{27}{ }^{28}$ Of the three patients reporting both symptoms at 18 months, two had a prior history of treatment for mental health problems; this may suggest that symptoms initially triggered by the $\mathrm{SAH}$, as by other traumas, are likely to persist over the long-term primarily in people with a pre-existing vulnerability to mental health problems. ${ }^{29} 30$

Although mean scores on the HADS and the BDI did not change significantly, remaining within normal limits, it is notable that clinical levels of HADS anxiety and depression were respectively three times and twice as likely to be reported by the SAH patients than by the matched controls. Similarly, eight of the SAH patients (16.3\%) had BDI scores over 18, consistent with at least a mildly depressive state; only three of these had a previous history of treatment for mental health problems. These rates of clinically significant mood disturbance, though elevated, are markedly lower than the $75 \%$ reported by Beristain et al $^{2}$ or the $30-50 \%$ suggested by Politynska et $a^{31}$ from a review of published studies. The reason for these disparities is unclear, though one possibility is that the patient groups had differential access to sources of post-discharge support. Pritchard et $a^{32}$ have recently highlighted the hiatus in care and support which frequently occurs following hospital discharge and have linked this to major psychosocial problems for SAH patients and their carers. Our research group is currently investigating this issue experimentally via a randomised controlled evaluation of post-discharge support from a nurse specialist.

When predictors of continued mood disturbance were explored statistically, prior mental health treatment accounted for $16 \%$ of the variance in mood outcome, and the presence of dysphasic symptoms 3 months after surgery for a further $9 \%$. None of the other variables entered into the model (age, gender, initial outcome grade (GOS $4 v 5$ ), impaired prose recall at 3 months, pre-SAH life stress, or preSAH physical health problems) increased explanatory power; indeed, the total variance explained was only $20 \%$ and the overall model fell just short of significance. Thus mood disturbance seen at this duration after SAH appears to be only in relatively small part attributable to pre-existing difficulties, with post-SAH cognitive impairments possibly serving as a small additional influence.

Perhaps surprisingly, the participants' observed elevated levels of dependence on others for activities and organisation and the low levels of productive employment were not associated with low mood. Thus, their reduced levels of activity and participation appear not either to result from or to contribute to dysphoria. This may reflect the anecdotal observations noted earlier, that for some patients, reductions in activity may in part represent positive lifestyle adjustments 
rather than a deterioration in personal autonomy. It is too simplistic for clinicians and researchers to assume that a decline in these aspects of social functioning necessarily represents a reduction in patients' quality of life; indeed, insofar as the absence of subjective distress indicates an acceptable quality of life, these data suggest that the road to recovery need not necessarily focus on promoting resumption of prior or even "normal" patterns of activity. In future research it would be valuable to ask participants directly about their perceived reasons for changes in their lifestyles, and about their future aspirations.

Fatigue, by contrast with mood, may be an important influence on outcome in this group. When, based on spontaneous reports of problematic fatigue at earlier followup points, we asked them to rate the severity of their fatigue at 18 months on a simple 3 point scale, a statistical association emerged with some of the psychosocial outcomes, particularly frequency of socialising and level of independence in self-organisation. The mechanisms underlying fatigue after brain injury are far from clear, and it may be that it is as much a consequence as a cause of failure to resume an active lifestyle: that is, failures to resume premorbid levels of activity might result in reduced fitness with consequent fatigue and reduced perceptions of competence. This is an interesting area for further investigation utilising more theoretically-conceived and well-validated measures of fatigue than the simplistic and unvalidated screening tool used here.

The trajectory and predictors of recovery observed within this "good neurological outcome" sample should not automatically be extrapolated to the broader SAH population, where the presence of more severe physical, sensory, or cognitive impairments may have a more significant bearing on psychosocial outcomes. Appropriate clinical interventions must depend on not only an accurate description of what has changed but on an understanding of the immediate and secondary psychological reactions that individual patients experience to their illness, hospitalisation, and subsequent symptomatology. Where patients' horizons become limited by health anxieties, fatigue, or a loss of confidence in their abilities, support and information from a knowledgeable professional after hospital discharge may on its own be sufficient to dispel inaccurate beliefs and prevent avoidable problems developing. In other cases, it may be appropriate to provide access to more intensive specialist interventions-for instance from a psychologist or occupational therapist- to overcome potentially debilitating mood disturbances or directly to assist the patient in re-engaging with activities of daily living and regaining their personal autonomy.

\section{ACKNOWLEDGEMENTS}

We are grateful to the Stroke Association for funding this study, to Judy Watkin (clinical nurse specialist) for facilitating contact with the patients, and to Alan Pickering for statistical advice. Finally, we thank all the participants, those with and those without subarachnoid haemorrhage, for their patience and time.

\section{Authors' affiliations \\ J Powell, J Heslin, Department of Psychology, Goldsmiths College, Lewisham Way, New Cross, London, SE14 6NW, UK \\ N Kitchen, R Greenwood, National Hospital for Neurology and Neurosurgery, Queen Square, London, WCIN, UK}

Competing interests: none declared

\section{REFERENCES}

1 Powell J, Kitchen N, Heslin J, et al. Psychosocial outcomes at 3 and 9 months after good neurological recovery from aneurysmal subarachnoid haemorrhage: predictors and prognosis. J Neurol Neurosurg Psychiatry 2002;72(6):772-81

2 Beristain X, Gaviria M, Dujony M, et al. Evaluation of outcome after intracranial aneurysm surgery: the neuropsychiatric approach. Surg Neurol 1996;45:422-9.

3 Mangold R, Wallenfang T. Posttraumatic neuropsychological and psychiatric disorders after subarachnoid haemorrhage. Nervenheilkunde 2000;19:32-7.

4 Horowitz M, Wilner N, Alvarez W. Impact of event scale: a measure of subjective stress. Psychosom Med 1979:41:209-18.

5 Buchanan KM, Elias $\amalg$, Goplen GB. Differing perspectives on outcome after subarachnoid haemorrhage: the patient, the relative, the neurosurgeon. Neurosurgery 2000;46:831-8.

6 Sonesson B, Liunggren B, Saveland $\mathrm{H}$, et al. Cognition and adjustment after late and early operation for ruptured aneurysm. Neurosurgery 1987;25:279-87

7 Sonesson B, Saveland $\mathrm{H}$, Liunggren B, et al. Cognitive functioning after subarachnoid haemorrhage of unknown origin. Acta Neurol Scand 1989:80:400-10.

8 Hillis $A E$, Anderson N, Sampath $P$, et al. Cognitive impairments after surgical repair of ruptured and unruptured aneurysms. I Neurol Neurosurg Psychiatry 2000;69:608-15.

9 Vilkki J, Holst P, Ohman J, et al. Social outcome related to cognitive performance and computed tomographic findings after surgery for a ruptured intracranial aneurysm. Neurosurgery 1990;26:579-85.

10 Ogden JA, Mee E, Henning M. A prospective study of psychosocial adaptation following subarachnoid haemorrhage. Neuropsychol Rehabil 1994:4:7-30.

11 Marquardt G, Nieubauer T, Schick U, et al. Long-term follow-up after perimesencephalic subarachnoid haemorrhage. J Neurol Neurosurg Psychiatry 2000;69:127-30.

12 Rodholm M, Starmark JE, Svensson E, et al. Astheo-emotional disorder after aneurysmal SAH: reliability, symptomatology and relation to outcome. Acta Neurol Scand 2001;103:379-85.

13 Hutter BO, Krietschmann-Andermahr I, Gilsbach JM. Health-related quality of life after aneurysmal subarachnoid haemorrhage: impacts of bleeding severity, computerized tomography findings, surgery, vasospasm, and neurological grade. J Neurosurg 2001;94:241-51.

14 Bond MR. Neurobehavioural sequelae of closed head injury. In: Grant I, Adams KM, eds. Neuropsychological assessment of neuropsychiatric disorders. New York: Oxford University Press, 1986.

15 Miller MA, Rahe RH. Life changes scaling for the 1990s. J Psychosom Res 1997;43:279-92.

16 Roberts CS, Severinsen C, Carraway C, Clark D, Freeman M, Daniel P. Life changes and problems experienced by young adults with cancer. J Psychosoc Oncol 1997; 15:15-25.

17 Wechsler D. Wechsler Memory Scale - revised. San Antonio, TX: Psychological Corporation, 1987.

18 Benton AL. Differential behavioural effects in frontal lobe disease. Neuropsychologia 1986;6:63-80.

19 Benton AL, Hamsher K de S. Multilingual Aphasia Examination. lowa City: University of lowa, 1976.

20 Powell J, Pickering A, Wyke $M$, et al. The effects of anti-hypertensive medication on learning and memory. Br J Clin Pharmacol 1993;35:105-13.

21 Zigmond AS, Snaith RP. The Hospital Anxiety and Depression Scale. Acta Psychiatr Scand 1983:67:361-70.

22 Beck AT. Beck Depression Inventory Manual. San Antonio, TX: Psychological Corporation, 1987.

23 Powell JH, Beckers K, Greenwood RJ. Measuring progress and outcome in community rehabilitation after brain injury with a new assessment measure the BICRO-39 scales. Arch Phys Med Rehabil 1998;79:1213-25.

24 Howell D. Statistical methods for psychology. 5th ed. Pacific Grove, CA: Duxbury, 2002.

25 McKenna P, Willison BA, Lowe D, et al. Cognitive outcome and quality of life one year after subarachnoid haemorrhage. Neurosurgery 1989;24:361-7.

26 Maurice-Williams RS, Willison JR, Hatfield R. The cognitive and psychological sequelae of uncomplicated aneurysm surgery. J Neurol Neurosurg Psychiatry 1991;54:335-40

27 Blanchard EB, Hickling EJ, Barton KA, et al. One-year prospective follow-up of motor vehicle accident victims. Behav Res Ther 1996;34:775-86.

28 Shalev AY, Freedman S, Peri T, et al. Prospective study of posttraumatic stress disorder and depression following trauma. Am J Psychiatry 1998; 155:630-7.

29 Paris J. Predispositions, personality traits and posttraumatic stress disorder. Harv Rev Psychiatry 2000;8:175-83.

30 McFarlane AC. Posttraumatic stress disorder: a model of the longitudinal course and the role of risk factors. J Clin Psychiatry 2000;61:15-23.

31 Politynska BE, Berrios GE, Lewko JL. Neuropsychiatric aspects of subarachnoid haemorrhage - a review. Neurol Psychiatr Brain Res, 1995;3:111-20.

32 Pritchard C, Foulkes L, Lang DA, et al. Psychosocial outcomes for patients and carers after aneurysmal subarachnoid haemorrhage. Br J Neurosurg $2001 ; 15: 4456-63$. 